University of Massachusetts Amherst

ScholarWorks@UMass Amherst

1990

\title{
Flow Injection Manifolds with Membrane Filters for Pre-concentration and Interference Removal by Precipitation Flow Injection Flame Atomic Absorption Spectrometry
}

Ebenezer Debrah

Loughborough University of Technology

C. E. Adeeyinwo

Loughborough University of Technology

S. R. Bysouth

Loughborough University of Technology

Julian Tyson

University of Massachusetts Amherst

Follow this and additional works at: https://scholarworks.umass.edu/chem_faculty_pubs

Part of the Chemistry Commons

\section{Recommended Citation}

Debrah, Ebenezer; Adeeyinwo, C. E.; Bysouth, S. R.; and Tyson, Julian, "Flow Injection Manifolds with Membrane Filters for Preconcentration and Interference Removal by Precipitation Flow Injection Flame Atomic Absorption Spectrometry" (1990). Analyst. 1392.

Retrieved from https://scholarworks.umass.edu/chem_faculty_pubs/1392 


\title{
Flow Injection Manifolds With Membrane Filters for Pre-concentration and Interference Removal by Precipitation Flow Injection Flame Atomic Absorption Spectrometry*
}

\author{
Ebenezer Debrah, + Christina E. Adeeyinwo, Stephen R. Bysoutht and Julian F. Tysont¥ \\ Department of Chemistry, University of Technology, Loughborough, Leicestershire LE11 3TU, UK
}

\begin{abstract}
The use of inexpensive, disposable, non-metallic filters in two manifolds for continuous precipitation, as a means of pre-concentration and matrix separation, is described. In one manifold, novel valve configuration and a simple timing device allowed the merging of sample and reagent streams, precipitation, and collection during the "load" part of the cycle. In the "inject" position, the stream switching flushed the dissolved precipitate through the filter. Copper was precipitated as the hydroxide and collected on a disposable cellulose acetate membrane filter. The degree of pre-concentration was shown to be proportional to the volume of sample passed. After sufficient precipitate had been collected, it was dissolved in a stream of hydrochloric acid. Each pre-concentration step was followed by a timed wash step before injection/ dissolution of the precipitate. For a sample volume of $11 \mathrm{ml}$, a pre-concentration factor of 12 was achieved. Calcium (10 $\left.\mathrm{mg} \mathrm{I}^{-1}\right)$ was shown to be selectively precipitated in the presence of an aluminium concentration of up to $1000 \mathrm{mg} \mathrm{l}^{-1}$, allowing the successful determination of calcium in the presence of aluminium, not normally achievable by atomic absorption spectrometry with an air - acetylene flame.
\end{abstract}

Keywords: Pre-concentration; precipitation; disposable membrane filters; flow injection; flame atomic absorption spectrometry

Although the various techniques of atomic spectrometry, in particular those of flame atomic absorption spectrometry (FAAS), electrothermal atomic absorption spectrometry (ETAAS) and inductively coupled plasma optical emission spectrometry (ICP-OES), are widely used in analytical methodology for the determination of elements (usually at trace concentrations), it is recognised that the techniques suffer from interference effects. ${ }^{1}$ In FAAS and ETAAS, both solute volatilisation effects and the overlap of the atomic line by a molecular band are causes of considerable inaccuracy. For ICP-OES, it is the problem of atomic spectral-line overlap that is perhaps the most severe interference effect, although in common with FAAS, considerable inaccuracies can arise from sample solution properties that affect the performance of the nebuliser and spray chamber. Much of the research concerned with these techniques is directed towards the goal of removing these interferences. In some respects, this area of research overlaps with the other major area of research in analytical atomic spectrometry, namely, the development of procedures concerned with obtaining detection limits of ever decreasing values.

A variety of approaches to these research goals have been proposed including, for plasma spectrometry, the use of "high solids" nebulisers, spectrometers with increased resolving power, and internal standardisation. The use of the mass spectrometer detector may be viewed as a means of reducing spectral interferences and improving detection limits. For ETAAS the emphasis has been on the separation of the matrix and analyte during the thermal pre-treatment stages in the furnace by various matrix (or analyte) modification procedures. Furnace design is evolving towards greater temporal and spatial uniformity of temperature with delayed atomisation through the use of platforms and probes, and improvements in the performance of background-correction devices are continually sought.

* Presented at SAC 89, the 8th SAC International Conference on Analytical Chemistry, Cambridge, UK, 30 July-5 August, 1989.

$\dagger$ Present address: Chemistry Department, University of Massachusetts, Amherst, MA 01003, USA.

$¥$ To whom correspondence should be addressed.
There has been less interest in the improvement of the performance of FAAS compared with ETAAS and ICP-OES/ mass spectrometry (MS) in recent years, with few research results being transferred to commercial availability apart from the slotted-tube atom retarder. Work on alternative aerosol generation and introduction devices and on various designs of atom trap are also being reported. Progress in all of the areas of research can be readily monitored by means of the relevant review literature. 2,3

From the earliest days of the application of atomic spectrometric techniques to the analysis of real samples, it has been recognised that there are benefits in using sample pre-treatment procedures whereby the analyte and matrix species are separated. One of the first of such procedures to be reported was liquid - liquid extraction, ${ }^{4}$ which, in common with most other procedures, has the benefit of producing an increase in sensitivity through pre-concentration and/or improved atomisation efficiency in addition to removing interfering matrix components. Other procedures for which a considerable literature exists include ion exchange, vaporisation and precipitation or coprecipitation. ${ }^{5}$ Procedures involving electrodeposition ${ }^{6}$ and foams ${ }^{7}$ have also been reported. With the exception of procedures that involve the conversion of mercury and some hydride-forming elements into volatile derivatives, none of these procedures has been developed in terms of a commercial apparatus directly coupled with the spectrometer. The most commonly used methodology for the other procedures has been in a manual, batch mode as the operations have proved to be difficult to automate, time consuming, labour intensive and prone to contamination and/or analyte loss.

Since the introduction of the concept of flow injection (FI) techniques in 1975, there has been a sustained interest in the application of such techniques to the development of automated, on-line sample pre-treatment procedures. ${ }^{8}$ Although the majority of FI techniques developed so far have been for methods in which the instrumental measurement has been of molecular species in solution (either by spectroscopic or electrochemical means), there has been a steady and growing interest in the use of FI techniques in conjunction with atomic spectrometry. Developments have been monitored in several reviews $^{9-14}$ and a recent book. ${ }^{15}$ It is clear from these that 
there is a growing interest in the use of FI techniques for the complete or partial removal of the matrix and for analyte pre-concentration, not only in conjunction with FAAS, but also with ICP-OES and ICP-MS. Most of the published work in this area so far has been concerned with the development of on-line liquid - liquid extraction or liquid - solid (usually chelating ion-exchange resin) extraction for use with FAAS Hence, the chemistry involved has been optimised for a single analyte. Recently, Santelli and co-workers have shown that it is possible to devise FI manifolds to exploit precipitation reactions for both sample pre-treatment ${ }^{16}$ and as the basis of indirect determinations. ${ }^{17}$ Again, the methods were developed for use in conjunction with a flame atomic absorption instrument. The precipitate was collected on a stainless-steel filter.

If the precipitation technique is to be applicable to spectrometric methods with multi-element capability and with greater detection power than FAAS, it is necessary to devise procedures involving non-specific reagents and non-metallic filter media. In this paper, the potential use of low-cost membrane filters is demonstrated for two precipitates of different characteristics, viz., copper hydroxide and calcium oxalate. The use of a novel manifold design, in which a confluence point and reaction zone are located in the loop of the "injection" valve, is also described.

\section{Experimental}

\section{Apparatus and Measurement Conditions}

\section{Pre-concentration}

A PU9100 atomic absorption spectrometer (Philips Scientific) was optimised for the determination of copper at a wavelength of $324.8 \mathrm{~nm}$, with use of an air - acetylene flame. The output was monitored by using an AR55 chart recorder (Philips Scientific). The manifold [Fig. 1(a)], containing P'TFE tubing ( $0.5 \mathrm{~mm}$ i.d.), was connected directly to the nebuliser of the spectrometer. Pump A (LKB) could be controlled externally and was connected to a timing device built in-house. This device also controlled the injection valve (PS Analytical). Pump B (Ismatec 840) ran continuously. A circuit diagram for the timing device is available from the authors. The filters used were cellulose acetate syringe filters with a pore size of $0.2 \mu \mathrm{m}$ and a diameter of $30 \mathrm{~mm}$ (Sartorius or Schleicher and Schuell). This configuration of the valve allows the eluent to flush all the manifold components downstream of the confluence point. As there is a danger of material adhering to the walls of the tubing and becoming lodged at the small discontinuities in the tube diameter, which occur at connections, this configuration has an advantage for precipitation reactions over the manifold design used previously for pre-concentration by liquid - solid extraction. 18 In that manifold, the eluent back-flushed the column, located in the loop of the injection valve, and did not flush the tubing between the confluence point and the valve.

\section{Interference removal}

The manifold [Fig. 1(b)] was constructed from $0.58 \mathrm{~mm}$ i.d. PTFE tubing. Samples of $38 \mu$ l were injected via a Rheodyne 6 port valve and passed down $26 \mathrm{~cm}$ of tubing before merging with the reagent. A mixing coil (length $50 \mathrm{~cm}, 0.58 \mathrm{~mm}$ i.d.) allowed formation of the precipitate before collection on the filter. The peristaltic pump (Gilson Minipuls 2) was fitted with PVC pump tubing of $2.0 \mathrm{~mm}$ i.d. Precipitates were collected on disposable nylon membrane filters of $0.45 \mu \mathrm{m}$ pore size and $25 \mathrm{~mm}$ diameter (Gelman). The manifold was connected to a Baird atomic spectrometer, optimised for the determination of calcium at $422.7 \mathrm{~nm}$ in an air - acetylene flame, via a T-piece to allow air compensation.

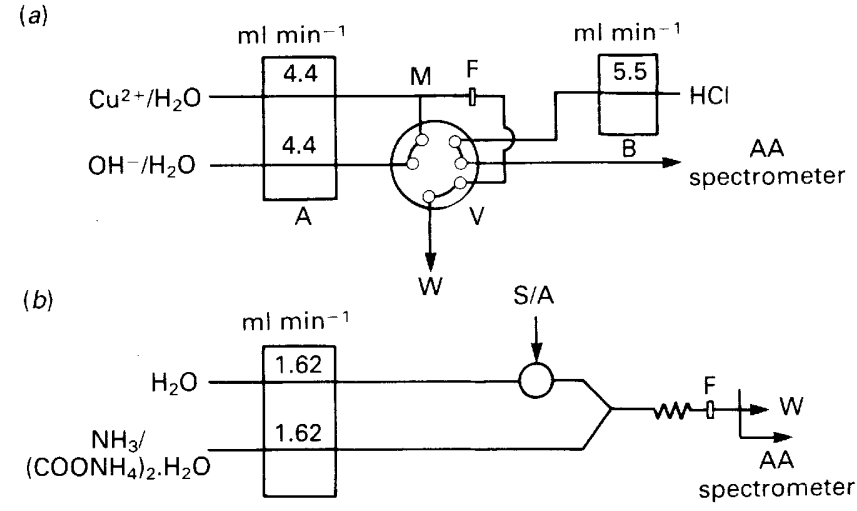

Fig. 1. (a) Flow manifold for the pre-concentration and determination of copper. F, Cellulose acetate filter; $W$, waste; $M$, merging point; A, LKB peristaltic pump; B. Ismatec peristaltic pump; and V, six-port injection valve (PS Analytical). (b) Flow manifold for the precipitation of calcium for removal of aluminium interference in the determination of calcium. S/A, Sample/dissolving acid; F, nylon filter; and $\mathrm{W}$, waste

\section{Reagents}

Copper standards were prepared by dilution of copper(II) nitrate (SpectrosoL, BDH) and acidified with $1 \%$ nitric acid (SpectrosoL, BDH). Sodium hydroxide solutions were prepared from analytical-reagent grade pellets (Fisons). Hydrochloric acid solutions were prepared by dilution of SpectrosoL-grade acid (BDH). Calcium solutions were prepared by dilution of stock $1000 \mathrm{mg} \mathrm{l}^{-1}$ standards (SpectrosoL, $\mathrm{BDH}$ ). Aluminium chloride (AnalaR, BDH) was added as an interferent. Oxalate solutions were prepared by dilution of ammonia solution (AnalaR, BDH) with the addition of ammonium oxalate (AnalaR, BDH). Reagent-grade water (obtained from a LiquiPure RG system, by reverse osmosis followed by ion exchange) was used throughout for dilution.

\section{Procedures}

\section{Pre-concentration}

On initiation of the pre-concentration cycle, the injection valve moves from the dissolution position to the pre-concentration position, and pump A, Fig. 1(a), pumps sample and hydroxide solution through the filter for a time set on the controller. After this time has elapsed, the pump stops and a light flashes to indicate to the operator that the sample and hydroxide solutions should be exchanged for a wash solution. When the wash cycle is initiated by the operator, the wash solution is pumped for the wash time ( $27 \mathrm{~s})$. After the wash time has elapsed, the filter is switched into the dissolving acid stream, and the collected hydroxide is dissolved and passes to the spectrometer. A second light then flashes to indicate that the system is ready for the next sample. All determinations are carried out in triplicate.

\section{Effect of hydroxide concentration}

A $0.1 \mathrm{mg} \mathrm{l}^{-1}$ copper(II) solution at a flow-rate of $4.4 \mathrm{ml} \mathrm{min}-1$ was merged with concentrations of hydroxide of $0.01,0.05$, $0.10,0.50,1.00$ and $2.00 \mathrm{~m}$ for $2.5 \mathrm{~min}$. The dissolving acid stream was $2 \mathrm{M} \mathrm{HCl}$ at a flow-rate of $5.5 \mathrm{ml} \mathrm{min}^{-1}$.

\section{Effect of dissolving acid concentration}

By using the same concentration of copper(II) and the same flow conditions as for the investigation of hydroxide concentration, the precipitate was dissolved in a number of $\mathrm{HCl}$ solutions of $0.002,0.05,0.10,0.50,1.00,1.50$ and $2.00 \mathrm{M}$. The concentration of hydroxide was $0.1 \mathrm{~m}$. 
Effect of sample flow-rate on efficiency of precipitation

Several $11-\mathrm{ml}$ aliquots of a $0.1 \mathrm{mg} \mathrm{^{-1 }}$ copper(II) solution were merged at flow-rates of $8.25,6.25,4.40,2.00$ and 1.25 $\mathrm{ml} \mathrm{min}-1$ with a $0.1 \mathrm{M}$ hydroxide solution pumped at the same flow-rates. The flow-rate of the dissolving acid stream, $2 \mathrm{~m}$ $\mathrm{HCl}$, was $5.5 \mathrm{ml} \mathrm{min}^{-1}$. The efficiency, assuming a linear calibration, was calculated as the ratio of absorbance to the mass of sample passed (which was determined by weighing the sample bottle before and after pre-concentration).

\section{Effect of dissolving acid flow-rate}

A $0.1 \mathrm{mg} \mathrm{I}^{-1}$ copper(II) solution was pre-concentrated by using $0.1 \mathrm{~m}$ hydroxide solution at a flow-rate of $4.4 \mathrm{ml} \mathrm{min}^{-1}$ for $2.5 \mathrm{~min}$. The flow-rate of the dissolving acid $(2 \mathrm{M} \mathrm{HCl})$ was

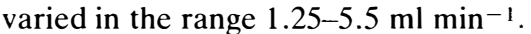

\section{Effect of mixing tube length}

The length of tube between the merging point and the filter was varied between 25 and $190 \mathrm{~cm}$. The tube was coiled to a radius of $0.5 \mathrm{~cm}$. The flow-rate of the sample was $4.4 \mathrm{ml} \mathrm{min}^{-1}$ and equalled that of the hydroxide solution. The flow-rate of

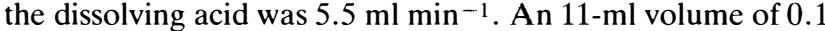
$\mathrm{mg} \mathrm{l}^{-1}$ copper(II) solution was merged with $0.1 \mathrm{M}$ hydroxide, and the dissolving acid was $2 \mathrm{M} \mathrm{HCl}$.

\section{Effect of length of tube between valve and spectrometer}

The length of the tube between the valve and the spectrometer was varied from 20 to $80 \mathrm{~cm}$. Precipitation was performed by using the same flow and chemical conditions as in the investigation of the effect of mixing tube length.

\section{Effect of sample volumes}

The volume of a $0.1 \mathrm{mg} \mathrm{l}^{-1}$ copper(II) solution was varied by sampling at a flow-rate of $4.4 \mathrm{ml} \mathrm{min}^{-1}$ for between 0.9 and 5.9 min. The $0.1 \mathrm{~m}$ hydroxide precipitant was merged at a flow-rate equal to that of the copper(II) solution.

\section{Sensitivity and detection limit}

Calibration was effected by pre-concentrating copper(II) solutions of $0,0.01,0.05,0.10,0.20$ and $0.50 . \mathrm{mg} \mathrm{l}^{-1}$ with $0.1 \mathrm{M}$ hydroxide as precipitant. Both the sample and precipitant streams were pumped at a flow-rate of $4.4 \mathrm{ml} \mathrm{min}^{-1}$, and the precipitate was dissolved in a stream of $2 \mathrm{M} \mathrm{HCl}$ at a flow-rate of $5.5 \mathrm{ml} \mathrm{min}^{-1}$. The same copper(II) solutions were aspirated in the conventional mode.

\section{Removal of aluminium interference}

The conditions for selective precipitation of calcium and subsequent dissolution by $\mathrm{HCl}$ with use of the manifold shown in Fig. 1(b) were optimised. Solutions consisting of $10 \mathrm{mg} \mathrm{l}^{-1}$ of calcium and various concentrations of aluminium were injected into a water carrier, flow-rate $1.62 \mathrm{ml} \mathrm{min}^{-1}$, and precipitated by merging with a solution of $50 \mathrm{mg} \mathrm{l}^{-1}$ of ammonium oxalate, flow-rate $1.62 \mathrm{ml} \mathrm{min}^{-1}$, in $0.05 \mathrm{M}$ ammonia. The precipitate was collected on the filter while the filtrate was allowed to pass to waste. The precipitate was subsequently dissolved in $0.5 \mathrm{M} \mathrm{HCl}$, before passing to the spectrometer.

\section{Results and Discussion}

\section{Copper Precipitation}

\section{Reagent concentration}

The results of the effect of hydroxide concentration on the precipitation of copper are shown in Fig. 2. It is observed that, with a hydroxide concentration between 0.1 and $0.5 \mathrm{M}$, the

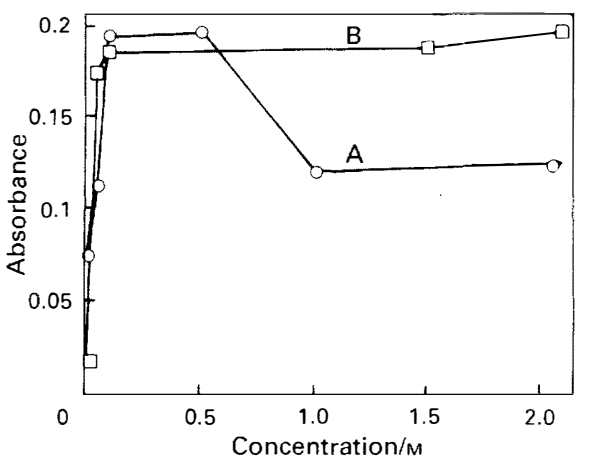

Fig. 2. Effect of A, hydroxide concentration and B, acid concentration on the signals for copper pre-concentration

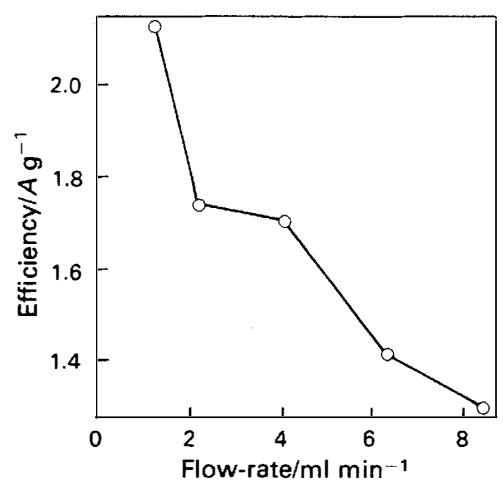

Fig. 3. Variation of pre-concentration efficiency with sample flowrate for copper. Reagent flow-rate was equal to sample flow-rate

absorbance signal is constant, but at higher or lower concentrations there is a decrease in signal. The decrease in signal at low concentrations of hydroxide could be due to the fact that at such levels there may not be sufficient hydroxide to precipitate the copper(II), while at higher concentrations of hydroxide the precipitate could be dissolved by the excess of hydroxide. ${ }^{19}$ The lower optimum concentration of hydroxide $(0.1 \mathrm{M})$ was, therefore, chosen for further experiments.

There was no significant difference in the signals obtained when the concentration of the dissolving acid was above $0.1 \mathrm{M}$. Below 0.1 M there was a significant reduction in the signal; up to $20 \%$ reduction for $0.05 \mathrm{M} \mathrm{HCl}$, indicating poor dissolution of the precipitate. This was confirmed by the peaks obtained at the lower acid concentrations, which were not as sharp as those for the $0.1 \mathrm{~m}$ acid.

\section{Optimisation of manifold}

The results for efficiency (Fig. 3) show that the precipitation reaction is most efficient at low flow-rates. However, as the gain in sensitivity at low flow-rates is not matched by the increase in analysis time, a flow-rate of $4.4 \mathrm{ml} \mathrm{min}^{-1}$ was chosen for the other experiments. At low acid flow-rates, the peaks obtained were broad, while at high flow-rates, the peaks were sharp and no tailing was observed (Fig. 4). A flow-rate of $5.5 \mathrm{ml} \mathrm{min}^{-1}$ was chosen, which gave the best peak shape and allowed reasonable sample throughput.

The peristaltic pump used to deliver sample and precipitant does not allow the flow-rate ratio to be varied from that set by the diameters of the pump tubing selected for each channel. Only two pump tubing sizes are available. The results in this study were obtained with equal-diameter pump tubing, but 


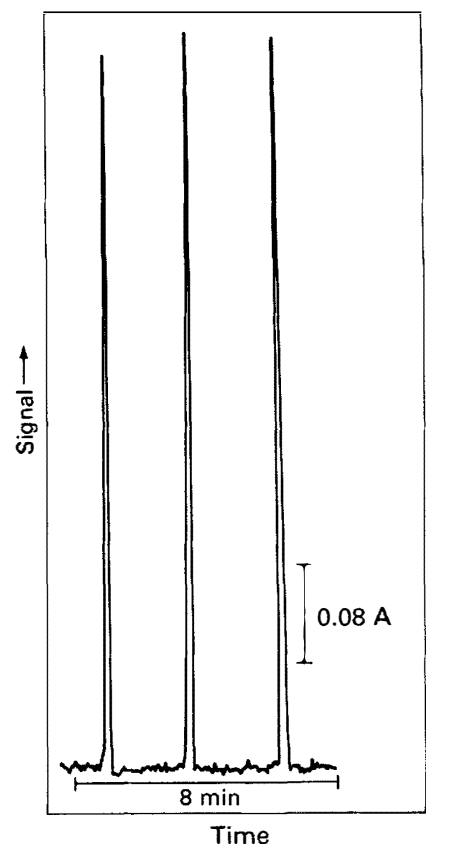

Fig. 4. Typical peaks for copper pre-concentration
Table 1. Mean peak absorbances for three replicate determinations of calcium in the presence of aluminium by precipitation with oxalate. Calcium concentration $=10 \mathrm{mg} \mathrm{l}^{-1}$

Alumin
concentr
$\mathrm{mg} \mathrm{l}$
20
400
700
1000

Standard deviation

0.002

0.005

0.010

0.005

0.002

reagent streams should not exceed $2 \mathrm{ml} \mathrm{min}^{-1}$. Increasing the sample volume from $18 \mu \mathrm{l}$ (to $38 \mu \mathrm{l}$ ) did not produce a proportional increase in peak height. A detection limit of 0.25 $\mu \mathrm{g} \mathrm{l}^{-1}$, obtained for analysis by precipitation under optimum conditions, is comparable to that obtainable with conventional aspiration. Results for the determination of calcium in the presence of various concentrations of aluminium are presented in Table 1. These show that the signal does not significantly decrease with increasing aluminium concentration and, therefore, that calcium is selectively precipitated by the reagent.

\section{Conclusion}

It has been shown that inexpensive, disposable, non-metallic membrane filters of at least two types can be used in continuous-flow precipitation manifolds to collect both gelatinous and crystalline material. A single switching/injection valve can be configured to control both the precipitation and elution reactions. The reagent concentrations and operating conditions could require further optimisation to achieve the best detection limits.

Financial support from Moses Annan for E.D., the Commonwealth Scholarship Commission for C.E.A., and the SERC for S. R. B. is gratefully acknowledged. The authors thank A. F. Bower for technical assistance in the design and construction of the timing device. injection valve and the nebuliser did not influence the peak height when $<40 \mathrm{~cm}$, whereas longer lengths increased dispersion and caused peak broadening.

\section{Effect of sample volume}

The absorbance signal increased proportionally to the volume of sample passed, up to $18 \mathrm{ml}$. At volumes $>18 \mathrm{ml}$, the plot curves gently. The curvature could be as a result of the non-linearity of the instrument response at this absorbance level, or the pre-concentration might, at this stage, become non-proportional. For concentrations within the linear part of the plot, it should be possible to generate a calibration graph with use of a single standard solution by pre-concentration of different volumes.

\section{Sensitivity and detection limit}

By examining the chart recording of the signal for the pre-concentrated standard of lowest concentration, a detection limit of $0.01 \mathrm{mg} \mathrm{l}^{-1}$ was attained, compared with 0.1 $\mathrm{mg} \mathrm{l}^{-1}$ for normal aspiration. The slope of the pre-concentration calibration graph was 1.47 , which, when compared with that obtained for normal aspiration, 0.13 , showed an increase in sensitivity by a factor of 12 for a sample volume of $11 \mathrm{ml}$.

\section{Removal of interference}

Optimisation of the conditions for the precipitation of calcium oxalate in the manifold [Fig. 1(b)] showed that for efficient precipitation, the flow-rate of the combined carrier and

\section{References}

1. Greenfield, S., Hieftje, G. M., Omenetto, N., Scheeline, A. and Slavin, W., Anal. Chim. Acta, 1986, 180, 69.

2. Dawson, J. B., Thorne, A. P., and Hill, S., J. Anal. At. Spectrom., 1990, 5, 151R.

3. Littlejohn, D., Jowitt, R., Shuttler, I., Sparkes, S. T., Tyson, J. F., and Walton, S. J., J. Anal. At. Spectrom., 1990, 5, 179R.

4. Cresser, M. S., "Solvent Extraction in Flame Spectroscopic Analysis," Butterworth, London, 1978, pp. 3-15

5. Minczewski, J., Chuastowska, J., and Dybczynski, R. "Separation and Preconcentration Methods in Inorganic Trace Analysis," Ellis Horwood, Chichester, 1982, pp. 37-96, and 283-502.

6. Long, S. E., and Snook, R. D., Analyst, 1983, 108, 1331.

7. Moody, G. J., and Thomas, J. D. R., Analyst, 1979, 104, 1.

8. Růžička, J., and Hansen, E. H., "Flow Injection Analysis," Second Edition, Wilcy, New York, 1988, pp. 323-364.

9. Tyson, J. F., Analyst, 1985, 110, 419.

10. Barnes, R. M., Spectroscopy, 1986, 1, 24.

11. Christian, G. D., and Růžička, J., Spectrochim. Acta, Part B, 1987, 42, 157

12. McLeod, C. W.. J. Anal. At. Spectrom., 1987, 2, 549

13. Tyson, J. F., Anal. Chim. Acta, 1988, 214, 57

14. Tyson, J. F. . Anal. Chim. Acta, 1990, 234, 3

15. Burguera, J. L., Editor, "Flow Injection Atomic Spectroscopy," Marcel Dekker, New York, 1989, pp. 103-292. 
16. Santelli, R. E., Gallego, M., and Valcarcel, M., J. Anal. At. Spectrom., 1989, 4, 547.

17. Montero, R., Gallego, M., and Valcarcel, M., J. Anal. At. Spectrom., 1988, 3, 725.

18. Bysouth, S. R., Tyson, J. F., and Stockwell, P. B., Anal. Chim. Acta, 1988, 214, 329.
19. Liptrot, G. F., "Modern Inorganic Chemistry," Mills and Boon, London, 1980.

Paper $9 / 04283 \mathrm{~K}$

Received October 6th, 1989 Accepted July 6th, 1990 